\title{
EVALUACIÓN DE UN NUEVO REGADÍO A LO LARGO DE 10 AÑOS: BALANCES HÍDRICOS, CALIDAD DEL RIEGO Y CONTAMINACIÓN POR SALES Y NITRATOS
}

\author{
Merchán, D. ${ }^{(1)}$, Causapé, J. ${ }^{(2)}$, Abrahao, . $^{(3)}$, García-Garizabal, I. ${ }^{(4)}$
}

\author{
1 Investigador Predoctoral, Instituto Geológico y Minero de España, C/ Manuel Lasala 44 \\ 9B, 50006 Zaragoza, d.merchan@igme.es \\ ${ }^{2}$ Científico Titular, Instituto Geológico y Minero de España, C/ Manuel Lasala 44 9ํㅗ, 50006 \\ Zaragoza, j.causape@igme.es \\ 3 Profesor, Universidad Federal de Paraíba, Cidade Universitária, Joao Pessoa, Paraíba, \\ 58051-970, Brasil, raphael@cear.ufpb.br \\ ${ }^{4}$ Investigador PROMETEO, Escuela Superior Politécnica del Litoral, Facultad de Ingeniería \\ en Ciencias de la Tierra, Km 30.5 Vía Perimetral, Campus Gustavo Galindo, Guayaquil, \\ Ecuador, ikerggarizabal@gmail.com
}

\section{Resumen}

La transformación al regadío es frecuente en zonas semiáridas a lo largo del mundo por sus numerosas ventajas, pero el regadío también es considerado como el principal consumidor de recursos hídricos y la principal fuente de contaminación difusa. En este trabajo se presenta una evaluación de la calidad del riego y del lixiviado de sales y nitratos en una cuenca hidrológica $\left(7,38 \mathrm{~km}^{2}\right)$ representativa de los regadíos presurizados de la cuenca del Ebro. El estudio abarca 10 años hidrológicos: antes (2004-2005), durante (20062008) y después (2009-2013) de la transformación de secano a regadío. Para este periodo, se realizaron balances hídricos y se obtuvieron indicadores de la calidad del riego a partir de éstos. Con la medida de la concentración de sales y nitratos en los diferentes componentes, se estimó la masa de estos contaminantes procedentes de la zona regable (352 ha), y se obtuvieron índices de contaminación. La eficiencia de riego alcanzó un 76,1\%, mientras que las pérdidas de eficiencia se debieron a la evaporación y arrastre del riego por aspersión $(13,5 \%)$ y al drenaje del riego $(10,4 \%)$. La eficiencia de riego aumentó $\left(1,05 \%\right.$ año $\left.{ }^{-1}\right)$ y la fracción de drenaje disminuyó $(0,95 \%$ año-1 $)$. Sin embargo, no se garantizó un incremento en la calidad del riego ya que el déficit hídrico también se incrementó $\left(0,95 \%\right.$ año $\left.{ }^{-1}\right)$ situándose en $17,8 \%$ al final del periodo de estudio. Tanto las masas exportadas como los índices de contaminación se incrementaron significativamente con la transformación al riego. Sin embargo, los valores obtenidos se encuentran por debajo del umbral de los regadíos considerados como más sostenibles. Mejoras en el manejo del riego, que tengan en cuenta la humedad del suelo y la velocidad del viento podrían incrementar de forma considerable la calidad del riego y disminuir el lixiviado y los índices de contaminación.

\section{1- Introducción y objetivos}

Durante las últimas décadas, la transformación de zonas de secano a agricultura de regadío es un proceso común en multitud de localizaciones por todo el mundo (FAO, 2003a). Las superficies transformadas experimentan incrementos en la productividad, así como una mayor estabilidad de las cosechas y diversidad en los cultivos. Aquellas cuencas hidrológicas en las que una alta proporción de su superficie se ve sometida a la transformación al regadío, en particular en zonas áridas o semiáridas, experimentan 
importantes cambios en su comportamiento hidrológico, tanto desde un punto de vista cuantitativo como en lo que respecta a la calidad del agua (e.g., Abrahão et al., 2011a, 2011b).

La progresiva escasez de agua que se constata en multitud de zonas del planeta pone de manifiesto la necesidad de evaluar los distintos usos del agua. En este sentido, la agricultura de regadío es reconocida como la principal consumidora de recursos hídricos del mundo (FAO, 2003b), lo que hace necesario la ejecución de estudios que evalúen la calidad del riego e incluyan, entre otros, indicadores de su eficiencia.

La eficiencia del riego a nivel de parcela depende de muchos factores concretos (tipo de suelo, diseño del sistema de riego, prácticas de riego, etc.). Por tanto, la eficiencia de riego varía de forma considerable tanto espacial como temporalmente, lo que hace difícil que unas "buenas prácticas agrícolas" sean de extensa aplicación, sino que estas prácticas deberán ser adaptadas al caso de estudio concreto (e.g., Hernández y Uddameri, 2010).

Por otro lado, la agricultura de regadío está reconocida como la principal fuente de contaminación difusa, por el alto lixiviado de sales y nitratos de zonas en riego, entre otros problemas ambientales (EPA, 1992). El lixiviado de sales es un requisito de la agricultura de regadío, ya que la acumulación de sales en los perfiles del suelo puede provocar daños en los cultivos, originando pérdidas de productividad e incluso forzando, en casos extremos, el abandono de las tierras. Sin embargo, los retornos de riego enriquecidos en sales pueden afectar la calidad de masas de agua, impactando tanto sistemas de abastecimiento humano como ecosistemas.

En lo que respecta a la contaminación por nitratos, es uno de los principales problemas de la agricultura en general por los riesgos asociados para la salud humana y para los ecosistemas (Sutton et al., 2011). La aplicación de fertilización nitrogenada normalmente no se corresponde con las necesidades de los cultivos (tanto en cantidad total como en distribución temporal a lo largo del ciclo del cultivo) lo que hace que haya abundante nitrógeno en el suelo para ser lixiviado por eventos de precipitación o riego que originen drenaje. El lixiviado de nitratos no sólo supone una pérdida de eficiencia en las inversiones de los agricultores, sino que además origina incrementos de concentración de nitratos en las masas de agua receptoras de los retornos de riego.

Aspectos relativos al balance hídrico, la calidad del riego o el lixiviado de sales y nitratos han sido objeto de numerosos estudios tanto en España como a nivel internacional. Sin embargo, este trabajo presenta una aproximación diferente ya que lo que se evalúa es la transformación de una zona de secano a regadío presurizado.

En este contexto, los objetivos de este trabajo son: (i) evaluar los cambios en el balance hídrico tras la transformación de secano a regadío presurizado; (ii) estudiar la evolución de la calidad del riego durante los años tras la transformación; (iii) evaluar los cambios en la contaminación por sales y nitratos originados por la transformación al riego presurizado; y (iv) comparar la contaminación que se origina con la de otras zonas regadas en la cuenca del Ebro.

\section{2- Zona de estudio}

La zona de estudio es la cuenca hidrológica del Barranco de Lerma (Ejea de los Caballeros, Zaragoza, $7,38 \mathrm{~km}^{2}$, Fig. 1), localizada en la margen izquierda del valle del Ebro. El $49 \%$ de su superficie fue transformada de secano a regadío de forma gradual entre los años 2006 y 2013, si bien los años que experimentaron mayores incrementos en la 
superficie regada fueron 2006 (36\% del área proyectada bajo riego), 2007 (76\%) y 2008 (90\%). Entre 2009 y 2013 se alcanzó el 100\% de la transformación.

La proporción de la cuenca que se decidió transformar viene condicionada principalmente por los materiales geológicos presentes en la misma (Fig. 1), correspondiéndose principalmente con materiales cuaternarios (gravas de matriz limosa) en los que se desarrollan suelos con baja pendiente y salinidad. En contraposición, la zona no transformada se sitúa sobre materiales terciarios (margas, arcillas, calizas margosas y yesos) en los que se desarrollan suelos con alta salinidad y pendiente.

Los principales cultivos presentes antes de la transformación eran el trigo y la cebada, sembrados en invierno y cosechados en junio o julio, y cuya producción dependía de las condiciones meteorológicas, con buenas cosechas en años húmedos. Entre los años 2003 y 2005 se produjo una interrupción en el cultivo de la tierra mientras tenían lugar los trabajos para la transformación al regadío (redistribución de las parcelas, construcción de caminos de acceso, instalación de la red principal de balsas y tuberías, etc.). Durante el periodo en regadío, el maíz se convirtió en el principal cultivo (44\% de la superficie), seguido por el cereal de invierno (19\%), el girasol y el guisante ( $9 \%$ cada uno) y el tomate $(6 \%)$. Se trata de regadíos presurizados, con la aspersión como principal sistema (93\%), y riego por goteo en el resto de la superficie, principalmente aplicado a hortícolas y frutales minoritarios (almendros).

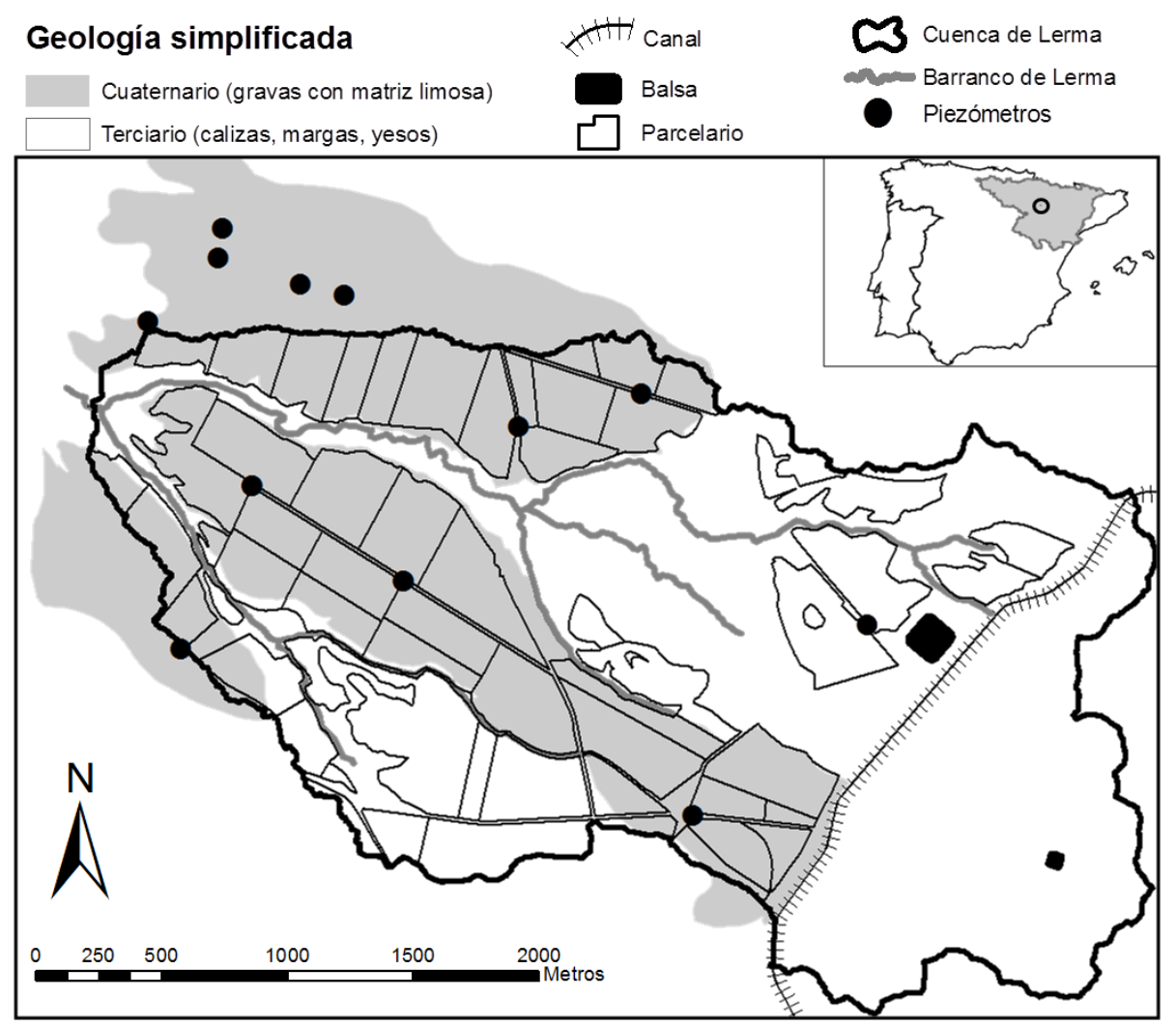

Figura 1. Zona de estudio con representación de la geología simplificada, canal y balsas de riego, parcelas regables, cuenca y barranco de Lerma, y red de piezómetros.

\section{3- Metodología}

La metodología de este trabajo consistió en la estimación de los diferentes componentes del balance hídrico, la obtención de indicadores de calidad del riego a partir de 
éstos, la estimación de las masas exportadas de contaminantes y la obtención de índices de contaminación por sales y por nitratos durante los años hidrológicos 2004-2013 (desde octubre de 2003 hasta septiembre de 2013). La metodología extendida está disponible en Causapé (2009), si bien aquí se presenta una síntesis de la misma.

\section{1- Balances hídricos}

A partir de datos meteorológicos obtenidos en fuentes oficiales (Red SIAR, Oficina del Regante del Gobierno de Aragón), de los datos de riego proporcionados por la comunidad de regantes y de una estimación de la capacidad de retención de agua disponible para las plantas (CRAD), se realizaron balances hídricos diarios en el suelo para cada una de las 55 parcelas agrícolas de regadío ubicadas en la zona de estudio, siguiendo la siguiente ecuación:

$$
(P+R)-\left(E T_{R}+D_{B A S}+P E A\right)=\Delta S
$$

donde la diferencia entre las entradas (precipitación, $\mathrm{P}$; riego, $\mathrm{R}$ ) y las salidas (evapotranspiración real, $\mathrm{ET}_{\mathrm{R}}$; drenaje del balance de agua del suelo, $\mathrm{D}_{\mathrm{BAs}}$; pérdidas por evaporación y arrastre de la aspersión, PEA) se corresponde con la variación en el almacenamiento en el suelo $(\Delta S)$.

Con posterioridad, se realizó el balance hídrico para el conjunto de la superficie regable de la cuenca, mediante la siguiente ecuación:

$$
(P+R+F H E)-\left(E T_{R}+B L+P E A\right)-(\Delta S+\Delta A)=\text { Error del balance }
$$

donde la diferencia entre las entradas (precipitación, $\mathrm{P}$; riego, $\mathrm{R}$; flujos hídricos entrantes generados en la zona no regable, FHE), las salidas (evapotranspiración real, $\mathrm{ET}_{\mathrm{R}}$; flujo saliente por el barranco de Lerma, BL; pérdidas por evaporación y arrastre de la aspersión, PEA) y el almacenamiento (en el suelo, $\Delta S$; en el acuífero, $\Delta \mathrm{A}$ ) se corresponde con el error del balance. En este tipo de estudios, errores del balance inferiores al $10 \%$ son considerados satisfactorios.

\section{2- Calidad del riego}

Una vez obtenido un balance de agua satisfactorio, se usaron los componentes del balance de agua en el suelo para obtener varios índices de calidad de riego (Causapé, 2009): eficiencia de riego (ER), fracción de drenaje del riego (FDR) y déficit hídrico (DH).

$$
\begin{gathered}
E R=100\left[1-\left(\left(D_{R}+P E A\right) / R\right)\right] \\
F D R=100\left(D_{R} / R\right) \\
D H=100\left[\left(E T_{C}-E T_{R}\right) / E T_{C}\right]
\end{gathered}
$$

donde: $D_{R}$ es el drenaje de agua de riego; PEA son las pérdidas por evaporación y arrastre del riego por aspersión; $R$ es el volumen de riego aplicado; y $E T_{C}$ y $E T_{R}$ son la evapotranspiración potencial y real respectivamente. La eficiencia de riego cuantifica el porcentaje de agua de riego que ha sido utilizado bien para satisfacer las necesidades de los cultivos o para aumentar el almacenamiento de agua en el suelo, esto es, que no se ha perdido como drenaje o evaporación y arrastre en el riego por aspersión. La fracción de drenaje del riego cuantifica el porcentaje de riego perdido como drenaje y está determinado por la cantidad de riego aplicado y el contenido de agua en el suelo cuando ocurre el evento de riego. Por último, el déficit hídrico evalúa en que medida las requerimientos hídricos del cultivo no han sido cubiertos. 


\section{3- Balance de sales y cuantificación del nitrato exportado}

Se realizaron mediciones o estimaciones del contenido de sales y nitratos en cada uno de los componentes del balance hídrico. En particular, se realizaron muestreos de agua de precipitación, riego y los flujos hídricos entrantes de la zona no regable a lo largo del periodo de estudio. Del mismo modo, se estimó el contenido en sales y nitratos del acuífero mediante una red de piezómetros disponible (Fig. 1). Las salidas de sales o nitratos por evapotranspiración o por pérdidas por evaporación y arrastre del riego por aspersión se consideraron nulas, ya que las concentraciones de sales y nitratos en estos componentes son despreciables. La concentración en las aguas de salida por el barranco de Lerma se estimó mediante una combinación de muestreos y de equipos de medida en tiempo real. A partir de las concentraciones y los volúmenes de cada componente (estimados en los balances hídricos) se obtuvieron los flujos de sales y nitratos.

En el caso del balance de sales, la diferencia entre entradas (precipitación, riego, flujos entrantes de la zona no regable), salidas (barranco de Lerma) y almacenamiento en el acuífero se corresponde con el resultado final del conjunto de procesos de disoluciónprecipitación. En el caso del nitrógeno, el objetivo no consistió en obtener un balance completo, sino cuantificar la masa exportada y la proporción de ésta que proviene de la precipitación, el riego y los flujos entrantes de la zona no regable. La diferencia entre la masa exportada y la cantidad procedente de riego, precipitación y zona no regable se corresponde con el resultado final del resto de procesos que afectan al balance de nitrógeno (fertilización, fijación, asimilación por las plantas, denitrificación, etc.).

En ambos casos, el resultado final de este apartado es la masa de sales o nitratos exportada por la zona regable de la cuenca de Lerma.

\section{4- Índices de contaminación}

Por último, dos índices propuestos por Causapé (2009) se obtuvieron para analizar en profundidad y poder comparar el impacto agro-ambiental producido: el índice de contaminación por sales (ICS) y el índice de contaminación por nitratos (ICN).

$$
I C S=D_{S} / C E_{N R} \quad I C N=D_{N} / N F
$$

donde $D_{S}\left(t\right.$ ha $\left.{ }^{-1} a n ̃ o^{-1}\right)$ y $D_{N}\left(\mathrm{~kg} \mathrm{ha}^{-1}\right.$ año $\left.^{-1}\right)$ son las masas exportadas de sales y nitrógeno en forma de nitrato, respectivamente. $\mathrm{CE}_{\mathrm{NR}}\left(\mathrm{dS} \mathrm{m}^{-1}\right)$ es la conductividad eléctrica del agua en el periodo sin riego, que sirve como indicador de la salinidad natural del sistema. NF ( $\mathrm{kg} \mathrm{ha}^{-1}$ año-1) representa las necesidades de fertilización de los cultivos presentes en la zona a evaluar.

De esta forma, las masas de contaminantes exportadas son corregidas por las condiciones naturales o sociales del sistema a evaluar, lo que posibilita una mejor comparación entre casos de estudio concretos. Regadíos que presentan valores de ICS menores de 2,0 [t ha-1 año-1]/[dS m$\left.{ }^{-1}\right]$ y de ICN menores de 0,2 se consideran entre los más sostenibles en lo que respecta a su lixiviado de sales y nitratos (Causapé, 2009). 


\section{4- Resultados y discusión}

\section{1- Balances hídricos}

Con la transformación, el riego se convirtió en la principal entrada de agua en la cuenca (60\% aproximadamente), sobrepasando los valores de precipitación en el cuarto año del estudio (2007). La evapotranspiración supuso la principal salida (73\%), seguida por las salidas a través del barranco de Lerma (22\%). Las pérdidas por evaporación y arrastre de la aspersión, si bien suponen un $5 \%$ de las salidas para todo el periodo de estudio, supusieron un $13,5 \%$ del agua de riego aplicada.

Los balances hídricos presentaron buenos resultados, con desbalances inferiores al $10 \%$ para la mayor parte de los años, y un desbalance del 1,2\% para todo el periodo de estudio, lo que se considera como satisfactorio y permite utilizar los datos obtenidos para evaluar la calidad del riego.

\section{2- Calidad del riego}

La eficiencia de riego alcanzó el $76,1 \%$, valores similares a los que se obtienen en otros estudios en regadíos presurizados del valle medio del Ebro. Las pérdidas de eficiencia se debieron a la evaporación y arrastre del riego por aspersión (13,5\%) y al drenaje (10,4\%). Se estimó un déficit hídrico del 17,8\%. Cabe destacar la ausencia de pérdidas por evaporación y arrastre en el riego por goteo, lo que pone de manifiesto la mayor eficiencia que este sistema de riego puede alcanzar.

Durante el periodo de estudio, la eficiencia de riego aumentó $\left(1,05 \%\right.$ año $\left.{ }^{-1}\right)$ y la fracción de drenaje disminuyó $(0,95 \%$ año-1 $)$. Sin embargo, no se garantizó un incremento en la calidad del riego ya que el déficit hídrico también se incrementó $(0,95 \%$ año-1), lo que sugiere que aún existe margen de mejora en la gestión del riego (Fig. 2).

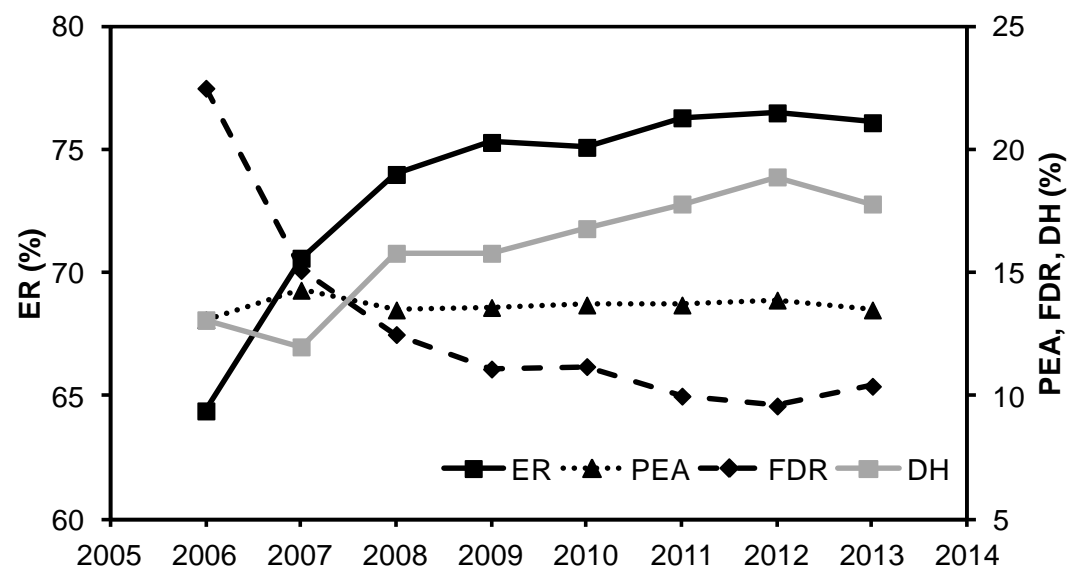

Figura 2. Evolución de la eficiencia de riego $(E R)$, pérdidas por evaporación y arrastre del riego por aspersión (PEA), fracción de drenaje del riego (FDR) y déficit hídrico (DH) durante el periodo en riego. Valores acumulados.

Por cultivos, el cereal de invierno y el girasol presentaron los mayores déficit hídricos (por encima del $20 \%$ ), como consecuencia de unas dotaciones de riego inferiores a las necesidades hídricas de estos cultivos, lo que probablemente haya afectado a su 
productividad. En el caso del maíz y del tomate, pese a tener dotaciones por encima de las necesidades hídricas, presentan déficits hídricos del $11 \%$ y $16 \%$ respectivamente, lo que sugiere un inadecuado calendario en lo que respecta a la aplicación del riego.

Con los datos expuestos, se concluye que para obtener un uso del agua óptimo y una mayor calidad en el riego se precisa de un adecuado diseño de calendarios de riego, esto es, que se ajusten de la mejor forma posible a las necesidades de los cultivos. Así mismo, el aumento de la proporción de riego por goteo o tener en cuenta factores como la humedad del suelo y la velocidad del viento es importante por su relevancia en la fracción de drenaje y en las pérdidas por evaporación y arrastre del riego por aspersión.

\section{3- Sales y nitrato exportado por la zona regable}

En lo relativo al lixiviado de sales, en condiciones de secano la zona de estudio exportó una media de $1,89 \mathrm{t} \mathrm{ha}^{-1} \mathrm{año}^{-1}$. Estos valores son inferiores a los que se constatan en otras zonas en secano de similar pluviometría pero mayor salinidad natural en el oeste de Australia (Salama et al., 1993). Tras la transformación al regadío, el lixiviado de sales se incrementó hasta valores medios de 3,51 $\mathrm{t} \mathrm{ha}^{-1} \mathrm{año}^{-1}$, valores en la parte inferior del amplio espectro de lixiviado de sales desde zonas regadas que aparece en la literatura (e.g., Duncan et al., 2008).

Por otro lado, el lixiviado de nitratos en secano fue de $11,4 \mathrm{~kg} \mathrm{ha}^{-1}$ año-1 de nitrógeno en forma de nitrato, algo inferior a lo que se obtiene en otras zonas en secano de la cuenca del Ebro con mayor pluviometría (Casalí et al., 2010). Estas cantidades se incrementaron hasta $30,8 \mathrm{~kg} \mathrm{ha}^{-1}$ año-1 de nitrógeno en forma de nitrato tras la transformación al regadío, valores de nuevo en la parte inferior del amplio espectro de lixiviado de nitratos en zonas regadas disponible en la literatura (e.g., Quemada et al., 2013).

Las comparaciones entre diversos estudios son difíciles por los distintos condicionantes naturales (salinidad del terreno) o antropogénicos (como el tipo de cultivos predominantes y las tasas de fertilización asociadas). Por ello se hace necesaria una herramienta que permita una mejor comparación teniendo en consideración dichos condicionantes, tal y como se propone con los índices de contaminación por sales y nitrato.

\section{4- Índices de contaminación}

Los índices de contaminación por sales y nitratos (ICS y ICN, respectivamente) se triplicaron con la transformación al regadío, alcanzando valores de 1,2 [t ha ${ }^{-1}$ año $\left.{ }^{-1}\right] /\left[\mathrm{dS} \mathrm{m}^{-1}\right]$ y 0,16 , respectivamente. Estos valores son similares a los que se obtienen para otros regadíos presurizados e inferiores a los que se obtienen para regadíos por inundación en diversos estudios realizados en la cuenca del Ebro (Tabla 1). Sin embargo, merece destacar como mejoras en el manejo del agua de riego en regadíos por inundación produce disminuciones significativas en los índices de contaminación, llevándolos en ocasiones a valores similares a los que se obtienen para regadíos presurizados. De hecho, para los estudios analizados se observa que existe relación entre la eficiencia de riego y el índice de contaminación por sales (Fig. 3). En el caso del índice de contaminación por nitratos, esta relación probablemente está enmascarada por aquellos factores relativos al manejo de la fertilización nitrogenada.

En el caso concreto de la cuenca de Lerma, a pesar de que los valores obtenidos para los índices de contaminación se encuentran dentro del umbral de los regadíos 
considerados como más sostenibles (ICS $<2,0\left[\mathrm{t} \mathrm{ha}^{-1}\right.$ año-1]/[dS m $\left.\mathrm{m}^{-1}\right]$ y ICN $<0,2$; Causapé 2009), sería aconsejable mejorar la gestión del riego de forma que se incremente el uso del agua y se disminuya el lixiviado de sales y nitratos.

Tabla 1. Eficiencia de riego (ER, \%), masa de sales exportada de la zona regada $\left(D_{s}, t h^{-1}\right.$ año-1 ${ }^{-1}$, conductividad eléctrica del punto de control durante el periodo sin riego (CE $\mathrm{NR}_{\mathrm{NR}} \mathrm{dS} \mathrm{m}^{-}$ $\left.{ }^{1}\right)$, índice de contaminación por sales (ICS, [t ha- $\left.\mathrm{año}^{-1}\right] /\left[\mathrm{dS} \mathrm{m} \mathrm{m}^{-1}\right]$ ), masa de nitrato en forma de nitrógeno exportada de la zona regada $\left(D_{N}, \mathrm{~kg} \mathrm{ha}^{-1} \mathrm{año}^{-1}\right)$, necesidades de fertilización (NF, $\mathrm{kg} \mathrm{ha}^{-1} \mathrm{año}^{-1}$ ) e índice de contaminación por nitratos (ICN, -) para varios distritos de riego en la cuenca del Ebro.

\begin{tabular}{|c|c|c|c|c|c|c|c|c|c|c|}
\hline $\begin{array}{l}\text { Zona de estudio } \\
\text { Distrito de riego }\end{array}$ & $\begin{array}{l}\text { Sistema } \\
\text { de riego }\end{array}$ & $\begin{array}{l}\text { Periodo } \\
\text { de estudio }\end{array}$ & $\begin{array}{l}\text { Área } \\
\text { (ha) }\end{array}$ & ER & Ds & $C E_{N R}$ & ICS & $D_{N}$ & NF & ICN \\
\hline $\begin{array}{c}\text { DXIXa } \\
\text { Bardenas I }\end{array}$ & $\begin{array}{l}\text { inundación } \\
\text { (mejorado)e }^{\mathrm{e}}\end{array}$ & $\begin{array}{c}2001 \\
2005-2008\end{array}$ & 100 & $\begin{array}{l}56 \\
83\end{array}$ & $\begin{array}{l}4,5 \\
1,3\end{array}$ & $\begin{array}{l}1,1 \\
1,1\end{array}$ & $\begin{array}{l}4,3 \\
1,2\end{array}$ & $\begin{array}{c}101 \\
51\end{array}$ & $\begin{array}{c}115 \\
76\end{array}$ & $\begin{array}{l}0,88 \\
0,67\end{array}$ \\
\hline $\begin{array}{l}\text { La Violadab } \\
\text { Monegros II }\end{array}$ & $\begin{array}{l}\text { inundación } \\
\text { (mejorado) }\end{array}$ & $\begin{array}{l}1996-1998 \\
2006-2008\end{array}$ & $4 \mathrm{k}$ & $\begin{array}{l}47 \\
58\end{array}$ & $\begin{array}{l}19,8 \\
10,2\end{array}$ & $\begin{array}{l}1,8 \\
2,0\end{array}$ & $\begin{array}{c}10,7 \\
5,0\end{array}$ & $\begin{array}{c}106 \\
22\end{array}$ & $\begin{array}{l}242 \\
115\end{array}$ & $\begin{array}{l}0,44 \\
0,19\end{array}$ \\
\hline $\begin{array}{l}\text { Lasesa }^{\mathrm{c}} \\
\text { Alto Aragón }\end{array}$ & $\begin{array}{l}\text { presurizado } \\
\text { maduro }\end{array}$ & 2010 & $1,4 \mathrm{k}$ & 76 & 0,5 & 1,5 & 0,3 & 49 & 288 & 0,17 \\
\hline $\begin{array}{l}\text { DIX \& DXId } \\
\text { Monegros II }\end{array}$ & presurizado & $\begin{array}{l}1997-1999 \\
1997-1998\end{array}$ & $\begin{array}{l}494 \\
470\end{array}$ & 80 & $\overline{14,0}$ & 8,4 & $\begin{array}{l}- \\
1,7\end{array}$ & $\begin{array}{l}18 \\
49\end{array}$ & $\begin{array}{l}225 \\
223\end{array}$ & $\begin{array}{l}0,08 \\
0,22\end{array}$ \\
\hline $\begin{array}{c}\text { Lerma } \\
\text { Bardenas II }\end{array}$ & $\begin{array}{l}\text { presurizado } \\
\text { reciente }\end{array}$ & $2009-2013$ & 352 & 76 & 4,4 & 3,5 & 1,2 & 41 & 267 & 0,16 \\
\hline
\end{tabular}

a García-Garizábal et al., 2012, 2014.

${ }^{b}$ Barros et al., 2012a, 2012b.

c Andrés y Cuchí, 2014.

d Tedeschi et al., 2001 (datos de sales), Cavero et al., 2003 (datos de nitratos).

e (mejorado) implica mejoras en la gestión del regadío (e.g., riego a la demanda en lugar de por turnos).

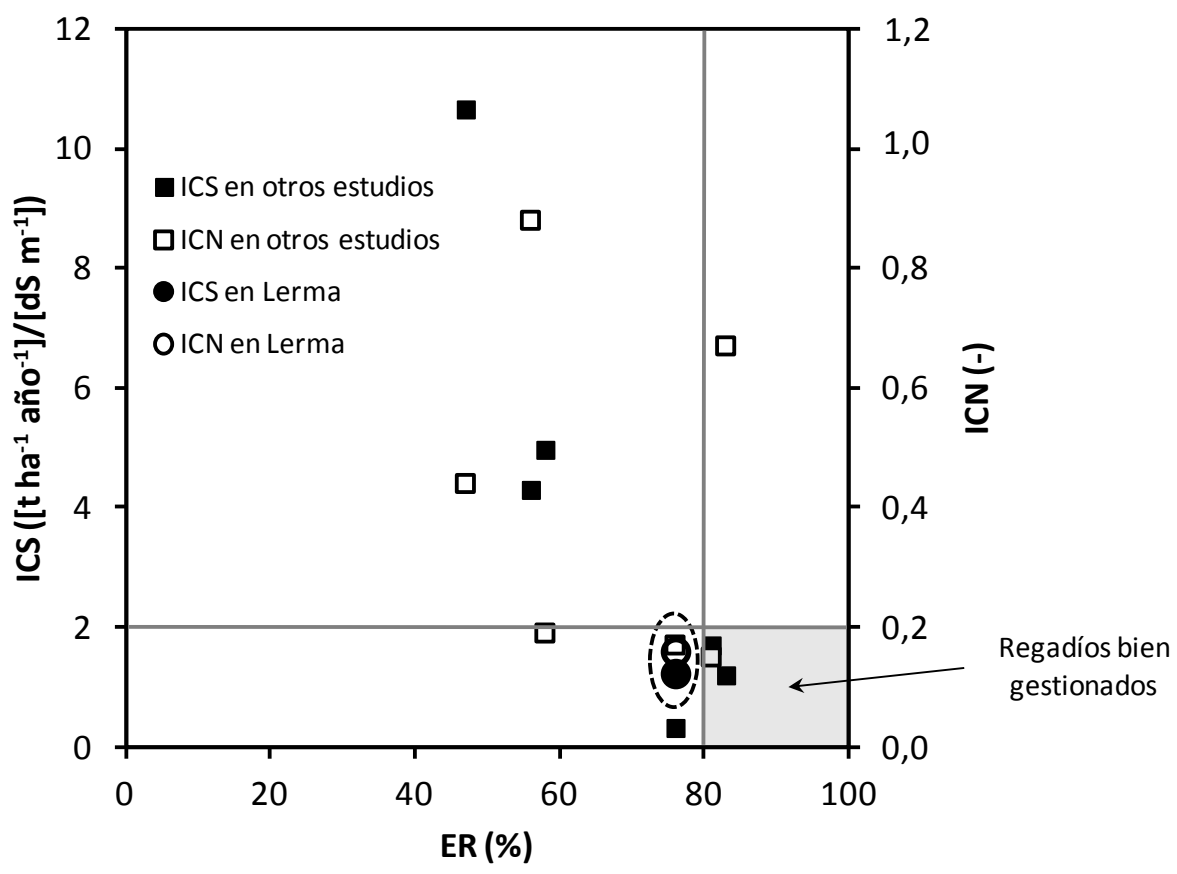


Figura 3. Eficiencia de riego (ER), índice de contaminación por sales (ICS) y por nitratos

(ICN) en diversas zonas de estudio en la cuenca del Ebro. Se destaca la zona de los regadíos más sostenibles en lo que respecta al lixiviado de sales y nitratos.

\section{5- Conclusiones}

La calidad del riego en la cuenca de Lerma es similar a la que se estima para otros regadíos presurizados de la cuenca del Ebro. Pese a que algunos de los indicadores mejoraron durante el periodo de estudio -incrementos en la eficiencia de riego y descensos en la fracción de drenaje-, no se garantiza una mejora en la calidad del riego debido al aumento observado en el déficit hídrico. Esto hace que sea necesario continuar con la mejora del manejo del riego, ajustando la aplicación del agua de riego a las necesidades hídricas de los cultivos y teniendo en consideración factores como la humedad presente en el suelo o la velocidad del viento, de forma que se pueda aumentar la eficiencia y disminuir el drenaje, las pérdidas por evaporación y arrastre de la aspersión, y el déficit hídrico.

Las masas de sales y nitrato exportadas por la zona regable se incrementaron significativamente con la transformación en riego, alcanzando valores similares a los que se obtienen en otros regadíos presurizados. Los índices de contaminación en la cuenca de Lerma, pese a estar por debajo del umbral de lo que se consideran regadíos más sostenibles, son susceptibles de mejorar debido al margen de mejora detectado en el manejo del riego. Medidas complementarias a la optimización del riego como mejorar el manejo de la fertilización o el uso de cultivos cubierta serían de gran utilidad para minimizar el lixiviado de contaminantes en zonas regables.

\section{Agradecimientos}

Este trabajo se ha financiado mediante el proyecto CGL2012-32395 (Ministerio de Economía y Competitividad con participación de fondos Feder - Unión Europea). Así mismo, ha contado con la colaboración de la Comunidad de Regantes ํㅡ XI de Bardenas. D. Merchán agradece la financiación a través de la ayuda BES2010-034124 (Ministerio de Economía y Competitividad) e I. GarcíaGarizábal ha contado con financiación a través del programa Prometeo (SENESCYT-Ecuador).

\section{Bibliografía}

Abrahão, R., Causapé, J., García-Garizábal, I., Merchán, D. (2011a). Implementing irrigation: Water balances and irrigation quality in the Lerma basin (Spain). Agricultural Water Management, 102, 97-104.

Abrahão, R., Causapé, J., García-Garizábal, I., Merchán, D. (2011b). Implementing irrigation: Salt and nitrate exported from the Lerma basin (Spain). Agricultural Water Management, 102, 105-112.

Andrés, R., Cuchí, J.A. (2014). Salt and nitrate exports from the sprinkler-irrigated Malfarás creek watershed (Ebro river valley, Spain) during 2010. Environmental Earth Science, 72(7), 2667-2682.

Barros, R., Isidoro, D., Aragüés, R. (2012a). Three study decades on irrigation performance and salt concentrations and loads in the irrigation return flows of La Violada irrigation district (Spain). Agriculture, Ecosystems and Environment, 151, 44-52.

Barros, R., Isidoro, D., Aragüés, R. (2012b). Irrigation management, nitrogen fertilization and nitrogen losses in the return flows of La Violada irrigation district (Spain). Agriculture, Ecosystems and Environment, 155, 161-171. 
Casalí, J., Giménez, R., Díez, J., Álvarez-Mozos, J., Del Valle de Lersundi, J., Goñi, M., Campo, M.A., Chahor, Y., Gastesi, R., López, J. (2010). Sediment production and water quality of watersheds with contrasting land use in Navarre (Spain). Agricultural Water Management, 97, 1683-1694.

Causapé, J. (2009). A computer-based program for the assessment of water-induced contamination in irrigated lands. Environmental Monitoring Assessment, 158, 307-314.

Cavero, J., Beltrán, A., Aragües, R. (2003). Nitrate exported in drainage waters of two sprinkler-irrigated watersheds. Journal of Environmental Quality, 32(3), 916-926.

Duncan, R.A., Bethune, M.G., Thayalakumaran, T., Christen, E.W., McMahon, T.A. (2008). Management of salt mobilization in the irrigated landscape - A review of selected irrigation regions. Journal of Hydrology, 351, 238-252.

EPA (Agencia de Protección Medioambiental de Estados Unidos, 1992). Managing non-point source pollution. Washington, DC: EPA, Office of Water.

FAO (Organización de las Naciones Unidas para la Alimentación y la Agricultura, 2003a). Unlocking the Water Potential of Agriculture. United Nations Food and Agriculture Organization, FAO, Rome, Italy.

FAO (2003b). World Agriculture Towards 2015/2030. A FAO Perspective. United Nations Food and Agriculture Organization, FAO, Rome, Italy.

García-Garizábal, I., Causapé, J., Abrahão, R. (2012). Nitrate contamination and its relationship with flood irrigation management. Journal of Hydrology, 442-443, 15-22.

García-Garizábal, I., Gimeno, M.J., Auqué, L.F., Causapé, J. (2014). Salinity contamination response to changes in irrigation management. Application of geochemical codes. Spanish Journal of Agricultural Research, 12(2), 376-387.

Hernández, E.A., Uddameri, V. (2010). Selecting agricultural best management practices for water conservation and quality improvements using Atanassov's Intuitionistic Fuzzy Sets. Water Resources Management, 24, 4589-4612.

Quemada, M., Baranski, M., Nobel-de Lange, M.N.J., Vallejo, A., Cooper, J.M. (2013). Metaanalysis of strategies to control nitrate leaching in irrigated agricultural systems and their effects on crop yield. Agriculture, Ecosystems and Environment, 174, 1-10.

Salama, R.B., Farrington, P., Bartle, G.A., Watson, G.D. (1993). Salinity trends in the wheatbelt of Western Australia: results of water and salt balance studies from Cuballing catchment. Journal of Hydrology, 145, 41-63.

Sutton, M.A., Howard, C.M., Erisman, J.W., Billen, G., Bleeker, A., Grennfelt, P., van Grisven, H., Grizzetti, B. (2011). The European Nitrogen Assessment: Sources, Effects and Policy Perspectives. Cambridge University Press, Cambridge.

Tedeschi, A., Beltrán, A., Aragüés, R. (2001). Irrigation management and hydrosalinity balance in a semi-arid area of the middle Ebro river basin (Spain). Agricultural Water Management, 49, 31-50. 